\section{MS3-O3 Processing XFEL Still Shots and Merging Multi-crystal Data}

Nicholas K. Sauter ${ }^{1}$, Iris D. Young ${ }^{1}$, Aaron S. Brewster ${ }^{1}$

1. Lawrence Berkeley National Laboratory

email: nksauter@lbl.gov

The ability to avoid radiation damage with femtosecond $\mathrm{X}$-ray pulses is a powerful argument for the use of X-ray free electron lasers (XFELs), with the important caveat that the observed diffraction consists entirely of partially recorded Bragg spots. Correcting the structure factor intensity to the full spot equivalent improves the quality of the final, merged data set that is created by mutually scaling hundreds or thousands of separate shots. However, several years after the initial XFEL results, efforts are still ongoing to produce optimal models of the spot partiality, with programs such as cctbx.xfel, DIALS, Prime, and ccpxfel each taking a slightly different approach. Data merging affects the final outcome, including the ability to phase with anomalous scattering data, and to discern high-resolution structural details. Examples will be shown from recent data collection at LCLS, including results from the new protein crystallography end station MFX.

Keywords: XFEL, diffraction

\section{MS3-O4 Data sets merging in serial crystallography}

Rangana S. Warshamanage ${ }^{1}$, Vincent Olieric ${ }^{1}$, Chia-Ying Huang ${ }^{2}$, Martin Caffrey ${ }^{2}$, Kay Diederichs ${ }^{3}$, Meitian Wang ${ }^{1}$

1. Swiss Light Source, Paul Scherrer Institute, CH 5232 Villigen, Switzerland

2. Membrane Structural and Functional Biology Group, School of Medicine and School of Biochemistry and Immunology, Trinity College, Dublin 2, Ireland

3. Fachbereich Biologie, Universität Konstanz, D-78457 Konstanz, Germany

email: rangana.warshamanage@psi.ch

In synchrotron serial crystallography experiments by the IMISX method micro-crystals in the lipid mesophase are sandwiched between thin cyclic olefin copolymer windows [1,2] and measured in situ with an X-ray microbeam (Figure 1). Small rotations of crystals are achieved by turning the whole plate mounted on the goniometer. These rotations allow one to record full intensities of reflections. In this geometry, it is difficult to collect a complete data set from a single crystal. A full data set can be recorded by merging many partial data sets from randomly oriented crystals. However, prior to merging isomorphous data sets must be identified. The criteria used for this purpose rely on the correlation between an individual data set and the rest of the data [2]. Data set selection is carried out iteratively. This method can be used not only to identify isomorphous data sets but also to identify the data frames compromised by radiation damage. In this presentation, details of the method and our recent results will be presented.

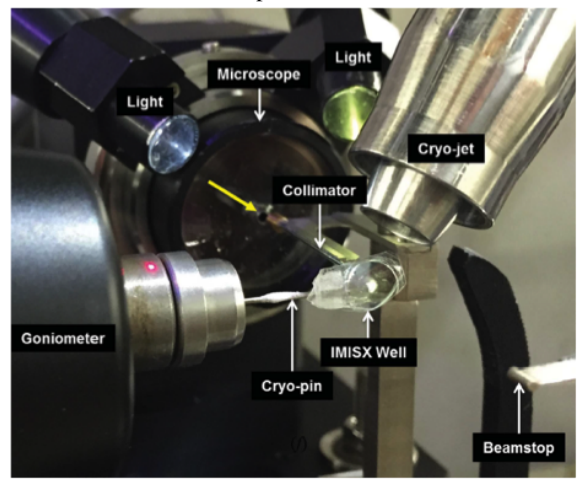

Figure 1. Experimental setup for IMISX data collection on beamline PXI (X06SA) at the Swiss Light Source.

Keywords: synchrotron serial crystallography, isomorphous data set, IMISX method 